\title{
Airway inflammation during stable and acutely exacerbated chronic obstructive pulmonary disease
}

\author{
K. Fujimoto, M. Yasuo, K. Urushibata, M. Hanaoka, T. Koizumi and K. Kubo
}

ABSTRACT: The aim of this study was to clarify the mechanism of increased airway inflammation during an acute exacerbation.

A total of 68 chronic obstructive pulmonary disease patients in a stable phase were enrolled and followed-up for 2-3 yrs. Inflammatory cells were analysed, and interleukin (IL)-8, neutrophil elastase, eotaxin, tryptase and RANTES (regulated on activation, normal T-cell expressed and secreted) were measured in sputum, both in a stable phase and during acute exacerbation.

Out of 68 patients, 30 (unstable group) developed an acute exacerbation and expectorated adequate sputum during exacerbation. Thirty-two patients (stable group) did not develop any exacerbation for 2-3 yrs. The number of neutrophils, lymphocytes and eosinophils, and the levels of IL-8, eosinophil cationic protein (ECP), eotaxin and tryptase in sputum obtained from patients in both groups during the stable phase were significantly higher than those from healthy nonsmokers. There were no significant differences in cell analysis and biomarkers between the two groups, but patients in the unstable group showed more severe airflow limitation. In the unstable group, total cells, lymphocytes, neutrophils and eosinophils, and IL-8, neutrophil elastase, ECP and RANTES levels were significantly increased during an exacerbation from values in a stable phase.

These findings suggest that exacerbation of chronic obstructive pulmonary disease may associate with additional increases in airway inflammation mediated by neutrophils, lymphocytes, eosinophils, interleukin-8 and RANTES.

\section{KEYWORDS: Acute exacerbation, airway inflammation, chemoattractant, eosinophils, neutrophils}

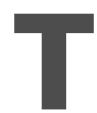
he prognosis for chronic obstructive pulmonary disease (COPD) is adversely affected by acute exacerbation, which increases mortality and morbidity, and causes an irreversible decline of pulmonary functions, thus possibly contributing to the development of serious illness [1, 2]. It has been suggested that the exacerbation may be associated with the increase in airway inflammation resulting from various causes, such as viral or bacterial infection, or environmental factors. AARON et al. [3] demonstrated that markers of airway neutrophilic inflammation increase at the time of acute COPD exacerbation; however, this acute inflammatory response appears to occur independently of a demonstrable viral or bacterial airway infection. BНоWмIK et al. [4] demonstrated that patients with more frequent exacerbations have higher baseline sputum interleukin (IL)- 6 and IL8 levels, and these cytokines may predict the frequency of future exacerbations. It has also been reported that not only neutrophils but also eosinophils and lymphocytes may participate in the increased airway inflammation during acute exacerbation $[5,6]$. However, the details of the mechanism of increase in airway inflammation during exacerbation remain obscure.

It has been suggested that, among various causes of acute exacerbation, respiratory tract infection due to a virus or bacteria may account for as much as, or even more than, $80 \%$ of COPD exacerbations, and that between one-third and one-half of all causes clearly are the result of viral infection [7-9]. It has been demonstrated that viral infection of airway epithelial cells induces various cytokines and chemokines, such as IL-8, eotaxin and RANTES (regulated on activation, normal T-cell expressed and secreted) [10, 11]. It has been suggested that these cytokines and chemokines play a role in airway neutrophilic and eosinophilic inflammation as chemoattractants in the pathogenesis of COPD [7]. Conversely, it has also been demonstrated that

\section{AFFILIATIONS}

First Dept of Internal Medicine, Shinshu University School of Medicine, Matsumoto, Japan.

\section{CORRESPONDENCE}

K. Fujimoto

First Dept of Internal Medicine Shinshu University School of

Medicine

3-1-1 Asahi

Matsumoto

390-8621

Japan

Fax: 81263363722

E-mail: keisaku@hsp.md.shinshu-

u.ac.jp

Received:

April 222004

Accepted after revision

December 212004 
mast cells increase in the airways of COPD cases [12], and that the concentration of tryptase degranulated from mast cells in sputum increases in COPD patients, with evidence of sputum eosinophilia [13]. Although the role of mast cell activation in COPD is still obscure, it has been suggested that activated mast cells in the airway may contribute to airway hypersecretion $[14,15]$.

For the current study, it was hypothesised that neutrophils and eosinophils, recruited by chemoattractants such as IL-8, RANTES and eotaxin, as well as mast cells, may contribute to the increase in airway inflammation during an acute exacerbation. To examine this hypothesis, inflammatory cells in sputum were examined, and the concentrations of IL-8, neutrophil elastase, tryptase, eosinophil cationic protein (ECP), eotaxin and RANTES in sputum obtained from patients with COPD, both in a stable phase and while exacerbated, were measured. The present authors also examined whether these airway inflammatory cells and biomarkers in sputum predict the frequency of exacerbations.

\section{MATERIALS AND METHODS Subjects}

A total of 68 consecutive patients with COPD, admitted to the current authors' outpatient clinic (Shinshu University Hospital, Matsumoto, Japan) from October 1998 to September 2001 and consistent with selection criteria, with adequate sputum obtained in a stable phase, were enrolled in this study. They had smoking-related COPD without $\alpha_{1}$-antitrypsin deficiency and a smoking history of $>30$ pack-yrs. COPD was diagnosed based on a clinical history of exertional dyspnoea, pulmonary function characterised by irreversible airway obstruction (forced expiratory volume in one second (FEV1)/forced vital capacity $<70 \%$ after inhalation of a short-acting $\beta_{2}$-agonist), lung hyperinflation, reduced gas transfer, and anatomic emphysema on high-resolution computed tomography (HRCT). Patients with any history of asthma, asthmatic symptoms such as coughing and wheezing at rest in a stable phase, as well as patients who had taken inhaled or oral steroids, or had suffered from respiratory tract infection or exacerbation during the preceding 3 months were excluded. The severity of COPD according to Global Initiative for Chronic Obstructive Lung Disease (GOLD) guidelines [16] was as follows: 14 patients in stage 1, 23 in stage 2, 20 in stage 3 , and 11 in stage 4 . A total of 12 patients had been receiving long-term oxygen therapy, 43 had been treated with regular inhalation of an anti-cholinergic agent and/or long-acting $\beta_{2^{-}}$ agonist, and 42 had been treated with a slow-releasing theophylline for $>6$ months before the study. Eleven normal healthy male volunteers ranging 48-76 yrs in age, with a mean age of $66 \mathrm{yrs}$, served as controls; all were nonsmokers, nonatopic, and showed no abnormalities in a pulmonary function test. The study was approved by the local research ethics committee, and all patients gave their informed consent.

\section{Study design}

A total of 68 patients with COPD in a stable phase were enrolled from the present authors' outpatient clinic from October 1998 to September 2001. During the first visit, a history of current illness was obtained, and a routine physical examination, routine laboratory examinations, and chest radiograph were performed. During the second visit, a chest HRCT and pulmonary function tests were examined, and induced sputum was obtained from the patients by inhalation of hypertonic saline. All patients were followed-up at the outpatient clinic every month for 2-3 yrs. During this time, if the patient felt worse and contacted the current authors during the observation period, they were asked to come to the outpatient clinic as soon as possible, and a history and physical examination were obtained, sputum was checked, and spirometry (Chest Graph HI-701; Chest Co. Ltd, Tokyo, Japan) and oxygen saturation by pulse oximetry were measured (Pulsox-24M; Teijin Ltd, Osaka, Japan) or arterial blood gas analysis was carried out. After that, it was diagnosed whether the patient had developed an exacerbation or not, according to the definition by ANTHONISEN et al. [17], and sputum was obtained from the patients as frequently as possible. The stable phase was determined as a patient's stable condition continuing for $\geqslant 3$ months with normal day-to-day variations and without any signs of airway infection. In the current study, however, exacerbations due to heart failure, pulmonary thromboembolism, pneumothorax, respiratory muscle fatigue, narcotic agents and apparent pneumonia were excluded from the analysis. The sputum samples obtained during the stable and exacerbated phase were analysed for total and differential cell counts, and assayed for ECP, neutrophil elastase, IL-8, eotaxin, tryptase and RANTES.

\section{Sputum collection and analysis}

The current authors collected sputum induced by the inhalation of hypertonic saline using general principles as previously described [18]. However, during an acute exacerbation, the inhalation of hypertonic saline can sometimes have more serious consequences. In such cases, spontaneous sputum was obtained. The method of sputum induction is briefly outlined as follows. Prior to the induction of sputum, all subjects inhaled a $\beta_{2}$-agonist, and $3.5 \%$ hypertonic saline was nebulised with an ultrasonic nebuliser (NE-V10B; Omron, Tokyo, Japan) at maximum output for a $10-\mathrm{min}$ period. If appropriate sputum could not be obtained, $4.5 \%$ hypertonic saline was nebulised for 5 -min periods. If the FEV1 dropped by $>10 \%$ from the postbronchodilator value, the concentration of saline was not increased. If the FEV1 decreased by $>20 \%$ or if troublesome symptoms occurred, the nebulisation was discontinued. The nebulisation was continued for $\geqslant 10 \mathrm{~min}$ and stopped after 15 min or earlier if $\geqslant 2 \mathrm{~mL}$ of a sputum sample of good quality was obtained. When either spontaneous or induced sputum was collected, the subjects were asked to rinse their mouths and throats, and then to try to cough up sputum into a sterile plastic dish. The sputum was then separated from contaminating saliva by macroscopic examination, and the mucus plug was removed from the dish to a sterile plastic container, after which the volume of the sample was determined. A small aliquot of the sample to be used for cell counting was overlaid with an equal volume of Hank's balanced salt solution (HBSS) containing $1 \mathrm{mM}$ dithiothreitol (Sigma Chemicals, Poole, UK) and incubated at $37^{\circ} \mathrm{C}$ for $15 \mathrm{~min}$. The incubated suspension was then washed twice with HBSS. After the residual mucous was removed by filtering the suspension with gauze, the eluent was available for total and differential cell counts. The total cell count, except for that of squamous cells, was determined with a standard haemocytometer, normalised for weight and 
expressed as cells $\times 10^{5} \cdot \mathrm{g}^{-1}$ wet weight sputum. Cell smears were prepared with a centrifuge (Autosmear; Sakura, Tokyo, Japan) and stained with May-Grünwald-Giemsa stain for the differential cell count, which was carried out in a blinded fashion. The slides were coded and 500 cells were counted for the differential leukocyte count. It was determined whether the sputum was adequate or not by referring to PIN et al. [19]. Furthermore, the salivary contamination was also assessed by the percentage of squamous cells in differential cell counts, excluding epithelial cells, in the slides. This was accepted as adequate if the percentage of squamous cells was $<20 \%$ in dithiothreitol-treated samples [18]. The results of the differential leukocyte counts were expressed as a percentage of nucleated cells, not including squamous and epithelial cells. An equal volume of normal saline was immediately added to the remaining sputum samples, which did not contain dithiothreitol, and then the samples were sonicated and centrifuged at $2,000 \times g$ for $10 \mathrm{~min}$. The supernatant was aspirated and frozen at $-80^{\circ} \mathrm{C}$. The measurements of ECP, tryptase, neutrophil elastase, IL-8, eotaxin and RANTES were delegated to Mitsubishi Kagaku Bio-Clinical Laboratories Inc. (Tokyo, Japan). ECP and tryptase were measured by fluoroenzyme immunoassay using UniCAP ECP and UniCAP Tryptase kits, respectively (Pharmacia Diagnostics, Uppsala, Sweden). Neutrophil elastase was measured with the latex agglutination method, using an Ecoline PMN elastase kit (Merck Co., Darmstadt, Germany). IL-8, RANTES and eotaxin were determined by enzyme immunoassay, using Quantikine Human RANTES and Eotaxin Immunoassay kits, respectively (R\&D Systems, Minneapolis, MN, USA).

\section{Data analysis}

The values shown in the text and tables are mean \pm SEM. The data distribution of the variables in the various groups was first assessed with Bartlett's test. When the data for the variables showed a normal distribution, they were compared with a one-way ANOVA, followed by multiple comparisons with the Tukey-Kramer method. When the data for the variables did not show a normal distribution, the variables were compared with the Kruskal-Wallis test, followed by multiple comparisons among groups with the nonparametric Tukey-Kramer method. The comparisons between the variables in a stable phase and during an exacerbation were performed with a paired t-test. The correlation between variables was examined by calculating Pearson's product correlation coefficient. A p-value $<0.05$ was considered significant for all statistical test results.

\section{RESULTS}

Among the enrolled 68 patients, 34 patients developed an acute exacerbation for 2-3 yrs. Out of these 34 patients, adequate sputum samples during exacerbation could be obtained from 30 patients (unstable COPD), and the remaining four patients dropped out because adequate sputum during the exacerbated period could not be obtained $(n=2)$ or their only one-time exacerbation during the observation period was missed $(n=2)$. The mean period before developing an acute exacerbation was $11.5 \pm 1.7$ months in 30 patients. Twenty-one of the 30 patients developed an acute exacerbation more than two times during the observation period, and the mean exacerbation rate was $1.2 \pm 0.2$ events $\mathrm{yr}^{-1}$. Among the

remaining 34 patients, 32 patients did not develop any acute exacerbation for 2-3 yrs (stable COPD) and two patients had dropped out during the observation period because of a lack of contact with the current authors' outpatient clinic. In this study, the current authors analysed sputum obtained in a stable phase from 32 stable patients and sputum obtained from 30 unstable patients both in a stable phase and during the first acute exacerbated phase. Their ages, severity and pulmonary function test from a stable phase are shown in table 1 . There were no significant differences in age, sex and smoking history; however, the FEV1 was significantly lower and residual volume (RV)/total lung capacity (TLC) was significantly higher in 30 patients who developed exacerbation compared with 32 patients who did not develop exacerbation. Furthermore, although there were no severe patients in stage 4 in the stable COPD group, in the unstable COPD group, nine out of 30 were classed as severe COPD in stage 4 . The 30 patients who developed exacerbation were significantly more severe compared with 32 patients who did not.

Spirometry showed a significant decrease in FEV1 values from the stable to the exacerbated stage $(1.29 \pm 0.08 \mathrm{~L}$ versus $0.99 \pm 0.08 \mathrm{~L} ; \mathrm{p}<0.01)$. According to the severity of exacerbation as defined by ANTHONISEN et al. [17], it was mild in 13 patients, moderate in six, and severe in 11 . However, only three patients needed hospitalisation, and none had to be

\section{TABLE 1 Characteristics and pulmonary function}

Stable COPD

\begin{tabular}{|c|c|c|}
\hline Age yrs & $68.2 \pm 1.4$ & $68.9 \pm 1.2$ \\
\hline Males/females & $31 / 1$ & $27 / 3$ \\
\hline BI pack-yrs & $47.4 \pm 4.2$ & $53.9 \pm 3.7$ \\
\hline \multicolumn{3}{|c|}{ Severity according to GOLD staging } \\
\hline Stage 1 & 10 & 4 \\
\hline Stage 2 & 10 & 12 \\
\hline Stage 3 & 12 & 5 \\
\hline Stage 4 & 0 & $9^{* *}$ \\
\hline VC \% pred & $97.6 \pm 3.7$ & $94.0 \pm 3.0$ \\
\hline FEV1 L & $1.51 \pm 0.14$ & $1.29 \pm 0.08$ \\
\hline FEV $1 / F V C \%$ & $49.4 \pm 2.3$ & $43.7 \pm 2.3$ \\
\hline FEV $1 \%$ pred & $63.0 \pm 4.5$ & $52.9 \pm 4.0 * *$ \\
\hline RV \% pred & $220.9 \pm 12.8$ & $248.5 \pm 13.7$ \\
\hline RV/TLC \% & $53.1 \pm 1.8$ & $58.4 \pm 1.7^{*}$ \\
\hline$D$ L,Co \% pred & $70.2 \pm 3.9$ & $66.2 \pm 4.9$ \\
\hline $\mathrm{Pa}_{1} \mathrm{O}_{2}$ Torr & $74.1 \pm 1.9$ & $70.0 \pm 1.9$ \\
\hline 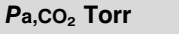 & $40.2 \pm 0.6$ & $40.6 \pm 0.7$ \\
\hline
\end{tabular}

Data are presented as mean \pm SEM. The data were obtained from 32 chronic obstructive pulmonary disease (COPD) patients who did not develop exacerbation (stable COPD) and 30 COPD patients who developed exacerbation more than once in the 2-3-yr follow-up period (unstable COPD). BI: Brinkman index; GOLD: Global Initiative for Chronic Obstructive Lung Disease; VC: vital capacity; \% pred: \% predicted; FEV1: forced expiratory volume in one second; FVC: forced vital capacity; RV: residual volume; TLC: total lung capacity; $\mathrm{LL}, \mathrm{CO}$ : carbon monoxide diffusing capacity of the lung; $\mathrm{Pa}_{\mathrm{a}} \mathrm{O}_{2}$ : arterial oxygen tension; $\mathrm{Pa}_{2} \mathrm{CO}_{2}$ : arterial carbon dioxide tension. ${ }^{*}: \mathrm{p}<0.05 ;{ }^{* *}: \mathrm{p}<0.01$ versus stable COPD. 
treated in the emergency clinic or had to be intubated. Increases in the severity of the symptoms of all patients were resolved by additional treatment with systemic corticosteroids and antibiotics, and, where needed, with a bronchodilator, but without hospitalisation.

\section{Cell analysis of sputum in a stable phase and during exacerbation}

In a stable phase, sputum total cell counts in the stable COPD group and numbers of neutrophils, lymphocytes and eosinophils in sputum from patients with both stable and unstable COPD groups were significantly higher than those in sputum from healthy nonsmokers (table 2). During exacerbation in the unstable COPD group, the total cell counts and absolute numbers of lymphocytes, neutrophils and eosinophils, as well as relative eosinophil counts, in sputum showed an additional significant increase over those in the stable phase. Figure 1 shows the changes in absolute neutrophil counts, and absolute and relative eosinophil counts in sputum from individual exacerbated patients with COPD. The concentrations of IL-8, ECP, eotaxin and tryptase in the supernatant of sputum from patients with both stable and unstable COPD groups in a stable phase were significantly higher than those in healthy nonsmokers (table 3). During the exacerbation, the levels of IL-8, neutrophil elastase, ECP and RANTES in sputum were significantly increased from those in the stable phase, whereas the eotaxin and tryptase levels did not show any significant changes.

\section{DISCUSSION}

It has been demonstrated that the numbers of eosinophils, neutrophils and CD3 lymphocytes in bronchial biopsies obtained during exacerbation increase compared with the corresponding numbers in those obtained during a clinically stable state from patients with mild COPD [20]. It was also demonstrated that the additional significant increase in the total cell counts and the numbers of lymphocytes, neutrophils and eosinophils in sputum corresponded to their exacerbations. The concentrations of ECP, a marker of eosinophil activation, in sputum obtained during an acute exacerbation also increased significantly compared with those in the stable state. These findings suggest that not only neutrophils but also eosinophils and lymphocytes may play a part in the increase in airway inflammation during acute exacerbation. Some reports have indicated that exacerbation of chronic bronchitis, defined by sputum colour, differs in the degree of bronchial and systemic inflammation, and that purulent exacerbation is related to bacterial infection and associated with increased neutrophilic inflammation [21]. In the purulent sputum of patients in the current study, the total number of recovered cells and neutrophils was higher than in nonpurulent sputum, but no such difference could be found in eosinophilic airway inflammation.

Levels of IL-8 and leukotriene $\mathrm{B}_{4}$, potent neutrophil chemoattractants, have been found to become elevated in sputum during acute exacerbation of COPD compared with their levels during a clinically stable stage [22]. In this study, the present authors also demonstrated a significant increase of IL-8 in sputum from patients with COPD in a stable phase compared with that in age-matched healthy nonsmokers, and an additional significant increase in IL-8 and neutrophil elastase during acute exacerbation, which corresponded to the increase in neutrophils in sputum. Neutrophil elastase degranulated from activated neutrophils can cause epithelial detachment and damage to mucociliary clearance and mucous hypersecretion [23]. These findings suggest that neutrophil recruitment

TABLE 2 Analysis of cells in sputum

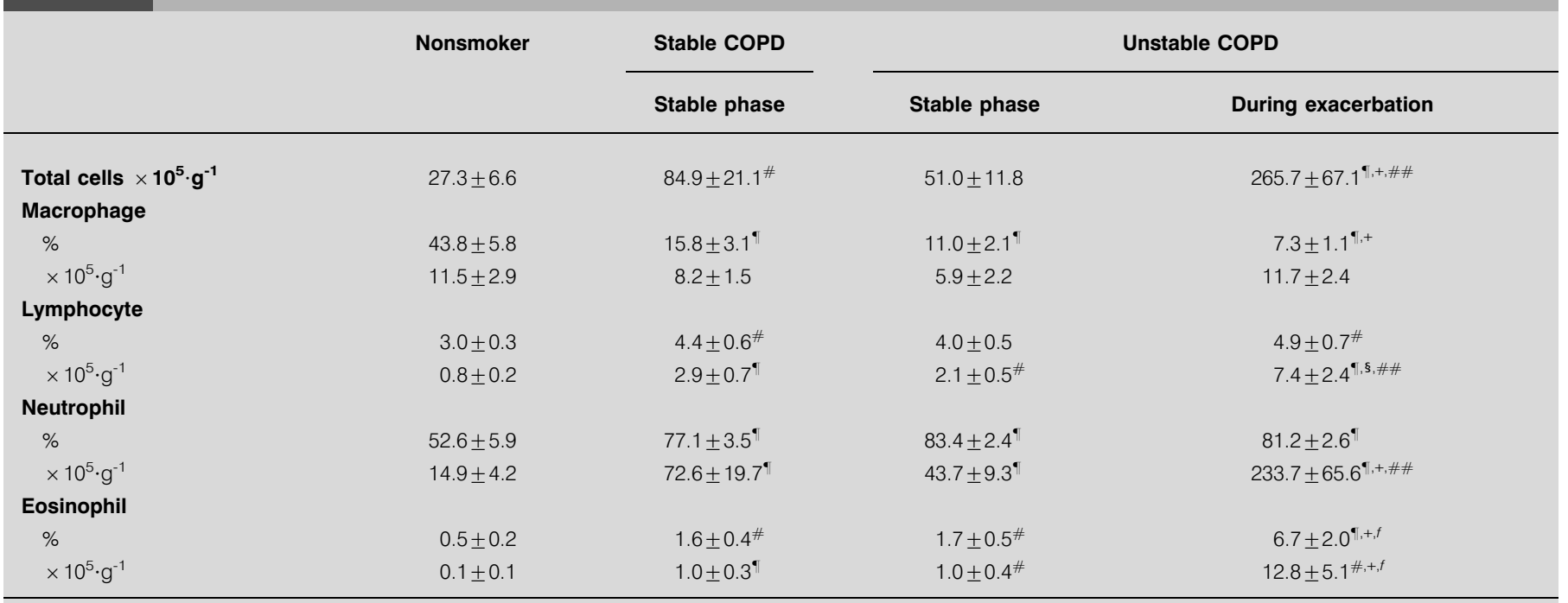

Data are presented as mean \pm SEM. Sputum was obtained from 11 nonsmokers, 32 chronic obstructive pulmonary disease (COPD) patients who did not develop exacerbation for 2-3 yrs (stable COPD), and 30 COPD patients who developed exacerbation (unstable COPD) in a stable phase and during exacerbation. ${ }^{*}: \mathrm{p}<0.05$ versus nonsmokers; ${ }^{\circ}: p<0.01$ versus nonsmokers; ${ }^{+}: p<0.05$ versus in a stable phase of stable COPD; ${ }^{\text {s. }}: p<0.01$ versus in a stable phase of stable COPD; ${ }^{f}: p<0.05$ versus in a stable phase of unstable COPD; \#\#: $p<0.01$ versus in a stable phase of unstable COPD. 

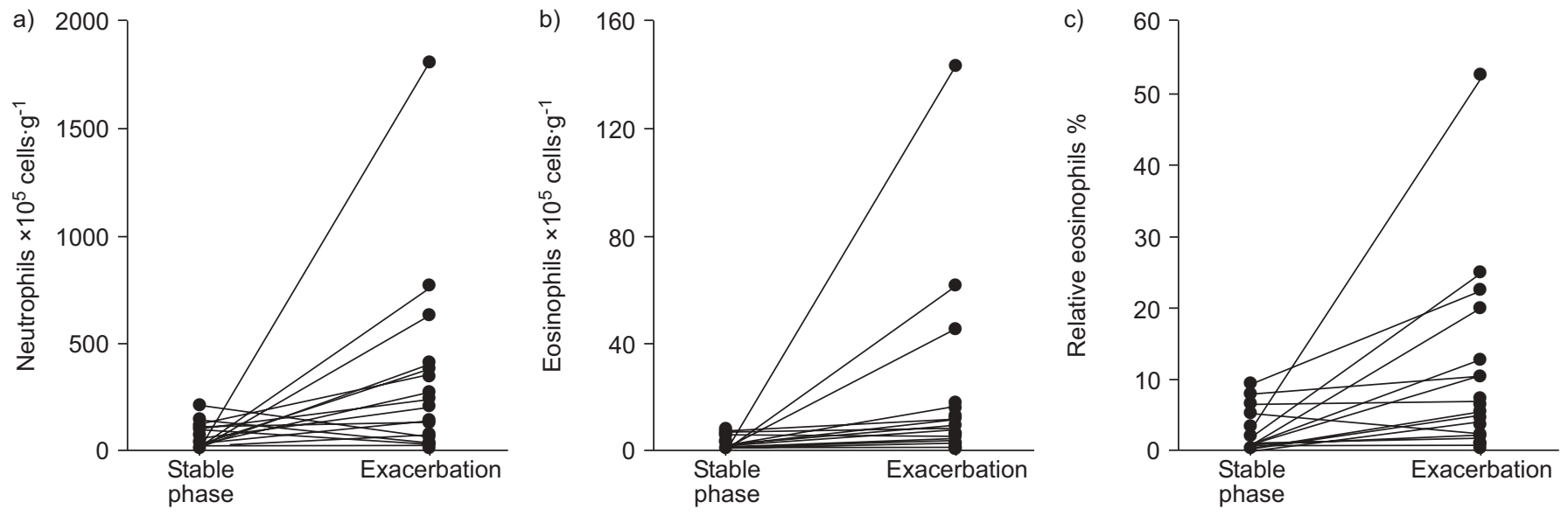

FIGURE 1. Changes in absolute neutrophil (a) and eosinophil (b) counts in $1 \mathrm{~g}$ of sputum and relative eosinophil (c) counts in sputum obtained in a stable phase and during an acute exacerbated phase from individual patients with unstable chronic obstructive pulmonary disease $(n=30)$. During exacerbation, the absolute neutrophil and eosinophil counts, as well as relative eosinophil counts, in sputum showed significant increases from those in the stable phase $(p<0.05)$.

and activation, in response to IL-8 as a chemoattractant, may play a role in the aggravation of airway inflammation during acute exacerbation.

ZHU et al. [6] have demonstrated that the numbers of lymphomononuclear cells expressing mRNA of eotaxin and RANTES, which are eosinophil chemoattractants, are significantly greater in smokers with chronic bronchitis than in healthy nonsmokers, and that the expression of RANTES in both the surface epithelium and subepithelial lymphomononuclear cells was further increased following acute exacerbation, whereas the expression of eotaxin did not show any significant change. A significant correlation between the number of eosinophilic granules type 2-positive eosinophils and that of RANTES-expressed lymphomononuclear cells was also found [6]. In the current study too, the eotaxin levels in sputum from patients with COPD in a stable phase were significantly higher than in sputum from age-matched nonsmokers, and RANTES showed a significant increase over the level in the stable phase, whereas eotaxin levels did not show any significant changes, similar to the histological findings by ZHU et al. [6]. These results suggest that eotaxin may participate in eosinophilic inflammation as eosinophil chemoattractants in the stable phase of COPD; conversely, RANTES may contribute to the aggravation of eosinophilic inflammation during acute exacerbation. The role of bacterial infections, once believed to be the main cause of COPD exacerbations, is now the subject of debate, since it is evident that much exacerbation in COPD is due to other causes, such as viral infections and environmental factors [24]. Infection of bronchial epithelial cells with human rhinoviruses results in the production of eotaxin and eotaxin-2, in addition to IL-8 and RANTES [11]. Respiratory syncytial virus also induces the production of IL-8 and RANTES from epithelial cell lines [10]. It is, thus, one possible mechanism that the neutrophil and eosinophil chemoattractants, IL-8 and RANTES, respectively, released from epithelial cells following viral infection may recruit these inflammatory cells and

\section{TABLE 3 Comparison of interleukin (IL)-8, neutrophil elastase (NE), eosinophil cationic protein (ECP), eotaxin, tryptase and} RANTES levels in sputum

\begin{tabular}{|c|c|c|c|c|}
\hline & Nonsmoker & $\begin{array}{l}\text { Stable COPD } \\
\text { Stable phase }\end{array}$ & Stable phase & During exacerbation \\
\hline $\mathrm{IL}-8 \mathrm{ng} \cdot \mathrm{mL}^{-1}$ & $1.16 \pm 0.18$ & $8.10 \pm 1.89^{\circ}$ & $4.79 \pm 1.37^{\#}$ & $22.73 \pm 7.06^{\oplus+, f}$ \\
\hline $\mathrm{NE} \boldsymbol{\mu g} \cdot \mathrm{mL}^{-1}$ & $0.36 \pm 0.13$ & $0.33 \pm 0.05$ & $0.64 \pm 0.18$ & 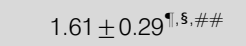 \\
\hline $\mathrm{ECP} \mu \mathrm{g} \cdot \mathrm{mL}^{-1}$ & $0.02 \pm 0.00$ & $0.47 \pm 0.16^{\circ}$ & $0.45 \pm 0.13^{\circ}$ & $2.50 \pm 0.76^{\bullet,+f}$ \\
\hline RANTES $\mathrm{pg} \cdot \mathrm{mL}^{-1}$ & $35.3 \pm 4.1$ & $41.5 \pm 4.0$ & $49.1 \pm 6.8$ & $137.9 \pm 38.2^{\#,+, f}$ \\
\hline
\end{tabular}

Data are presented as mean \pm SEM. Sputum was obtained from 12 nonsmokers, 32 chronic obstructive pulmonary disease (COPD) patients who did not develop exacerbation for 2-3 yrs (stable COPD), and 30 COPD patients who developed exacerbation (unstable COPD) in a stable phase and during exacerbation. ${ }^{\#}: \mathrm{p}<0.05$ versus nonsmokers; ${ }^{\circ}: p<0.01$ versus nonsmokers; ${ }^{+}: p<0.05$ versus in a stable phase of stable COPD; ${ }^{\varsigma}: p<0.01$ versus in a stable phase of stable COPD; ${ }^{f}: p<0.05$ versus in a stable phase of unstable COPD; \#\#: $p<0.01$ versus in a stable phase of unstable COPD. 
increase airway inflammation during acute exacerbation. Conversely, increased tryptase levels in the sputum from COPD during a stable state were also detected when compared with those in nonsmokers, although metachromatic cells in sputum were not evaluated. However, no significant association with sputum eosinophilia could be demonstrated, as has been reported by Louis et al. [13]. The elevated tryptase levels did not show any further increase during acute exacerbation. Although the role of mast cell activation in COPD remains obscure, mast cells in the airway may not appear to contribute to the further increase in airway inflammation during acute exacerbation.

BHOWMIK et al. [4] demonstrated that patients with more frequent exacerbations have higher baseline sputum IL-6 and IL-8 levels, and that IL-6 levels, but not IL-8, were further increased during exacerbations compared with stable conditions, whereas there were no significant increases in airway inflammatory cells during their acute exacerbation. They concluded that these cytokines may predict the frequency of future exacerbations. In the current study, when stable, the FEV1 was significantly lower and RV/TLC was significantly higher in 30 unstable COPD patients compared with 32 stable COPD patients. It has been demonstrated that the exacerbation frequency is associated with the severity of COPD [25]. However, there were no significant differences in cell analysis and biomarkers in sputum obtained during a stable phase between the two groups. Although IL-6 was not measured, it could not be demonstrated that the patients with more frequent exacerbations have higher baseline IL-8 in sputum. In the current study, mild COPD patients in stage 1 and 2 comprised $58.1 \%$ of all subjects and the mean FEV1 was $58 \%$ predicted. However, in the East London study [4], the enrolled COPD patients were moderate to severe and their mean FEV1 was $39.5 \%$ predicted; that is, the subjects had more severe COPD than those in the current study. Furthermore, the frequent exacerbation group was defined as the patients with $\geqslant 3$ exacerbations $\cdot \mathrm{yr}^{-1}$, and they developed exacerbation more frequently when compared with the patients with unstable COPD in this study whose exacerbated rate was $1.2 \pm 0.2$ events $\cdot \mathrm{yr}^{-1}$. There is some evidence that exacerbations are more frequent in those with regular sputum production [26]. In the present study population, when stable, most of the patients $(81 \%)$ did not have increased sputum and did not show regular sputum production, whereas $56 \%$ of patients had daily sputum in the East London study [4]. Furthermore, 21 of the patients were current smokers $(36.8 \%)$ and 51 of the patients were on inhaled steroids in the East London study [4], but most of the subjects in the current study were ex-smokers, current smokers were $<1 \%$ and none of subjects had taken inhaled or oral steroids. The smoking history and anti-inflammatory treatment may largely affect the exacerbation and airway inflammation. From these findings, the present authors speculated that the difference in results from the East London study [4] may be due to the differences in COPD severity, frequency of exacerbation, smoking history and steroid therapy. Also, the methodological difference may influence the result. BHOWMIK et al. [4] used daily diary cards and the monitoring of peak expiratory flow to evaluate exacerbations without missing any events, which may contribute to the difference in exacerbated frequency.
In conclusion, many inflammatory cells, such as lymphocytes, neutrophils, eosinophils and mast cells, and mediators, such as interleukin-8, eotaxin and tryptase, may be implicated in airway inflammation in chronic obstructive pulmonary disease in a stable phase. Of these inflammatory cells and mediators, eosinophils, as well as neutrophils, may also participate in the additional aggravation of airway inflammation during acute exacerbation of chronic obstructive pulmonary disease, and that interleukin- 8 and RANTES may play an important role in recruiting neutrophils and eosinophils into the airway during exacerbation.

\section{REFERENCES}

1 McCrory DC, Brown C, Gelfand SE, Bach PB. Management of acute exacerbations of COPD: a summary and appraisal of published evidence. Chest 2001; 119: 1190-1209.

2 Seemungal TA, Donaldson GC, Bhowmik A, Jeffries DJ, Wedzicha JA. Time course and recovery of exacerbations in patients with COPD. Am J Respir Crit Care Med 2000; 161: 1608-1613.

3 Aaron SD, Angel JB, Lunau M, et al. Granulocyte inflammatory markers and airway infection during acute exacerbation of chronic obstructive pulmonary disease. Am J Respir Crit Care Med 2001; 163: 349-355.

4 Bhowmik A, Seemungal TA, Sapsford RJ, Wedzicha JA. Relation of sputum inflammatory markers to symptoms and lung function changes in COPD exacerbations. Thorax 2000; 55: 114-120.

5 Saetta M. Airway inflammation in chronic obstructive pulmonary disease. Am J Respir Crit Care Med 1999; 160: S17-S20.

6 Zhu J, Qiu YS, Majumdar S, et al. Exacerbations of bronchitis: bronchial eosinophilia and gene expression for interleukin-4, interleukin-5, and eosinophil chemoattractants. Am J Respir Crit Care Med 2001; 164: 109-116.

7 White AJ, Gompertz S, Stockley RA. Chronic obstructive pulmonary disease. 6: The aetiology of exacerbations of chronic obstructive pulmonary disease. Thorax 2003; 58: 73-80.

8 Wedzicha JA. Exacerbations: etiology and pathophysiologic mechanisms. Chest 2002; 121: Suppl. 5, 136S-141S.

9 Greenberg SB, Allen M, Wilson J, Atmar RL. Respiratory viral infections in adults with and without chronic obstructive pulmonary disease. Am J Respir Crit Care Med 2000; 162: 167-173.

10 Noah TL, Wortman IA, Becker S. The effect of fluticasone propionate on respiratory syncytial virus-induced chemokines release by a human bronchial epithelial cell line. Immunopharmacology 1998; 39: 193-199.

11 Papadopoulos NG, Papi A, Meyer J, et al. Rhinovirus infection up-regulates eotaxin and eotaxin-2 expression in bronchial epithelial cells. Clin Exp Allergy 2001; 31: 1060-1066.

12 Grashoff WF, Sont JK, Sterk PJ, et al. Chronic obstructive pulmonary disease: role of bronchiolar mast cells and macrophages. Am J Pathol 1997; 151: 1785-1790.

13 Louis RE, Cataldo D, Buckley MG, et al. Evidence of mastcell activation in a subset of patients with eosinophilic 
chronic obstructive pulmonary disease. Eur Respir J 2002; 20: 325-331.

14 Pesci A, Rossi GA, Bertorelli G, Aufiero A, Zanon P, Olivieri D. Mast cells in the airway lumen and bronchial mucosa of patients with chronic bronchitis. Am J Respir Crit Care Med 1994; 149: 1311-1316.

15 Nadel JA. Role of mast cell and neutrophil proteases in airway secretion. Am Rev Respir Dis 1991; 144: S48-S51.

16 Global Initiative for Chronic Obstructive Lung Disease. Global strategy for the diagnosis, management and prevention of chronic obstructive pulmonary disease. NHLBI/WHO workshop report. Bethesda, National Heart, Lung and Blood Institute. April 2001: update of the management sections. www.goldcopd.com. Date last updated: July 12003.

17 Anthonisen NR, Manfreda J, Warren CP, Hershfield ES, Harding GK, Nelson NA. Antibiotic therapy in exacerbations of chronic obstructive pulmonary disease. Ann Intern Med 1987; 106: 196-204.

18 Fujimoto K, Kubo K, Yamamoto H, Yamaguchi S, Matsuzawa Y. Eosinophilic inflammation in the airway is related to glucocorticoid reversibility in patients with pulmonary emphysema. Chest 1999; 115: 697-702.

19 Pin I, Gibson PG, Kolendowicz R, et al. Use of induced sputum cell counts to investigate airway inflammation in asthma. Thorax 1992; 47: 25-29.
20 Saetta M, Di Stefano A, Maestrelli P, et al. Airway eosinophilia in chronic bronchitis during exacerbations. Am J Respir Crit Care Med 1994; 150: 1646-1652.

21 Gompertz S, O'Brien C, Bayley DL, Hill SL, Stockley RA. Changes in bronchial inflammation during acute exacerbations of chronic bronchitis. Eur Respir J 2001; 17: 1112-1119.

22 Crooks SW, Bayley DL, Hill SL, Stockley RA. Bronchial inflammation in acute bacterial exacerbations of chronic bronchitis: the role of leukotriene B4. Eur Respir J 2000; 15: 274-280.

23 Nadel JA. Role of neutrophil elastase in hypersecretion during COPD exacerbations, and proposed therapies. Chest 2000; 117: Suppl. 2, 386S-389S.

24 Saetta M, Turato G, Maestrelli P, Mapp CE, Fabbri LM. Cellular and structural bases of chronic obstructive pulmonary disease. Am J Respir Crit Care Med 2001; 163: 1304-1309.

25 Burge S, Wedzicha JA. COPD exacerbations: definitions and classifications. Eur Respir J 2003; 21: Suppl. 41, S46-S53.

26 Vestbo J, Prescott E, Lange P. Association of chronic mucous hypersecretion with FEV1 decline and chronic obstructive pulmonary disease morbidity. Copenhagen City Heart Study Group. Am J Respir Crit Care Med 1996; 153: 1530-1535. 